\title{
ON THE DVORETZKY-ROGERS THEOREM
}

\author{
by FUENSANTA ANDREU
}

(Received 9th April 1983)

The classical Dvoretzky-Rogers theorem states that if $E$ is a normed space for which $l^{1}(E)=l^{1}\{E\}$ (or equivalently $l^{1} \otimes_{\varepsilon} E \simeq l^{1} \otimes_{\pi} E$ ), then $E$ is finite dimensional (see [12] p. 67). This property still holds for any $l^{p}(1<p<\infty)$ in place of $l^{1}$ (see [7] p. 104 and [2] Corollary 5.5). Recently it has been shown that this result remains true when one replaces $l^{1}$ by any non nuclear perfect sequence space having the normal topology (see [14]). In this context, De Grande-De Kimpe [4] gives an extension of the DevoretzkyRogers theorem for perfect Banach sequence spaces.

In this paper, we show that the Dvoretzky-Rogers theorem holds for any echelon space of order $p(1<p<\infty)$ or order $(p, q)$ (see [1]). We also give a characterization of the nuclearity for echelon spaces of order $(p, q)$.

\section{Preliminaries}

All classical notations and properties concerning locally convex spaces and sequence spaces are taken from [9]. We follow [12] for nuclearity and [10] for tensor products.

We fix the following notations: We denote by $\mathbb{K}$ the field of real or complex numbers, by $\mathbb{N}$ the set of positive integers. Given $\alpha$ and $\beta$ in $\mathbb{K}$ we define $(\alpha ; \beta)$ as 0 if $\beta=0$ and $\alpha / \beta$ if $\beta \neq 0$. We write $|\alpha ; \beta|$ instead of $|(\alpha ; \beta)|$. If not specified, in what follows $E$ is a separated locally convex topological vector space over the field $\mathbb{K}$ with topological dual $E^{\prime}$. The spaces $c_{0}$ and $l^{p}(1 \leqq p<\infty)$ will represent the classical Banach spaces, whose definitions and properties can be seen in [9]. If $E$ is a vector space, $\omega(E)$ is the vector space of all sequences in $E$ and $\phi(E)$ is the subspace of $\omega(E)$ of all sequences $x=\left(x_{j}\right)$ which have finitely many non-zero $x_{j}$. If $E$ is the field $\mathbb{K}$, we write $\omega$ and $\phi$ instead of $\omega(\mathbb{K})$ and $\phi(\mathbb{K})$ respectively. Given $E$ and $F$ locally convex spaces, the symbol $E \simeq F$ has the following meaning: $E$ and $F$ are equal as vector spaces and the identity mapping is a topological isomorphism between them. If $\lambda$ is a sequence space, we define

$$
\lambda^{\alpha}=\left\{y=\left(y_{j}\right) \in \omega: \sum_{j=1}^{\infty}\left|x_{j} y_{j}\right|<\infty \quad \text { for all } \quad\left(x_{j}\right) \in \lambda\right\} .
$$

If $\lambda=\left(\lambda^{\alpha}\right)^{\alpha}$, then $\lambda$ is called a perfect sequence space. Given a perfect sequence space $\lambda$ whose topology is defined by the seminorms $\left\{p_{i}, i \in I\right\}$ and $(E,\|\|)$ a normed space, we consider the following generalized sequence spaces (see [3], [11] and [13])

$$
\lambda[E]=\left\{x=\left(x_{j}\right) \in \omega(E):\left(\left\langle x_{j}, u\right\rangle\right) \in \lambda \text { for all } u \in E^{\prime}\right\}
$$


provided with the $\varepsilon$-topology defined by the seminorms:

$$
\varepsilon_{i}(x)=\sup \left\{p_{i}\left(\left\langle x_{j}, u\right\rangle\right): u \in U^{0}\right\}, \quad i \in I,
$$

$U^{0}$ being the polar set in $E^{\prime}$ of the closed unit ball $U$ of $E$. If $x=\left(x_{j}\right) \in \omega(E)$, the sections of $x$ are defined by

$$
x^{(n)}=\sum_{j=1}^{n} x_{j} e_{j}
$$

for every $n \in \mathbb{N}, e_{i}$ being the sequence $\left(\delta_{i j}\right)$. The subspace of $\lambda[E]$ of all the elements $x$ such that $\left\{x^{(n)}\right\}$ converges to $x$ for the $\varepsilon$-topology is denoted by $\lambda(E)$, and we consider it endowed with the induced topology. Finally,

$$
\lambda\{E\}=\left\{x=\left(x_{j}\right) \in \omega(E):\left(\left\|x_{j}\right\|\right) \in \lambda\right\} .
$$

provided with the $\pi$-topology defined by the seminorms:

$$
\pi_{i}(x)=p_{i}\left(\left\|x_{j}\right\|\right), \quad i \in I .
$$

If $\lambda=l^{1}$, the former spaces are studied in [12]. The same notations and definitions will be used if $\lambda$ is a space of double sequences, having in mind that in this case the sections $x^{(n)}$ of $x=\left(x_{i j}\right)$ are defined by

$$
x^{(n)}=\sum_{i, j=1}^{n} x_{i j} e_{i j}
$$

for every $n \in \mathbb{N}, e_{i j}$ being the double sequence whose coordinates are null save the $(i, j)$ coordinate which has value one.

\section{The Dvoretzky-Rogers Theorem for echelon spaces of order $p$}

Let $\left\{a^{(r)}=\left(a_{j}^{(r)}\right)\right\}$ be a sequence of elements of $\omega$ satisfying:

(i) $a_{j}^{(r)}>0$ for all $r, j \in \mathbb{N}$

(ii) $a_{j}^{(r)} \leqq a_{j}^{(r+1)}$ for all $r, j \in \mathbb{N}$.

If $p$ is a real number with $p \geqq 1$, we denote by $\lambda_{p}$ the echelon space of order $p$ defined by the steps $\left\{a^{(r)}\right\}$ (see [9] and [15]), i.e.,

$$
\lambda_{p}=\left\{x=\left(x_{j}\right) \in \omega: \sum_{j=1}^{\infty}\left|x_{j} a_{j}^{(r)}\right| p<\infty \text { for all } r \in \mathbb{N}\right\},
$$

endowed with the topology defined by the seminorms:

$$
\|x\|_{r}=\left(\sum_{j=1}^{\infty}\left|x_{j} a_{j}^{(r)}\right|^{p}\right)^{1 / p}, \quad r=1,2, \ldots
$$


Analogously $\lambda_{\infty}$ and $\lambda_{0}$ denote the echelon spaces of order infinite and of order zero, respectively, defined by the steps $\left\{a^{(r)}\right\}$ with its usual topology (see [5] and [15]). It is well known (see [15]) that $\lambda_{p}$ is a nuclear space if and only if given a positive integer $r$ there is a positive integer $k>r$ such that

$$
\sum_{j=1}^{\infty}\left(a_{j}^{(r)} ; a_{j}^{(k)}\right)<\infty
$$

By the former result and the Grothendieck-Pietsch criterion (see [8]), we get:

Proposition 2.1. If $p$ is a real number with $p>1, \lambda_{p}$ is a nuclear space if and only if given a positive integer $r$ there is a positive integer $k>r$ such that

$$
\sum_{j=1}^{\infty}\left(a_{j}^{(r)} ; a_{j}^{(k)}\right)^{p}<\infty
$$

By [12] page $99, \lambda_{1}$ is a nuclear space if and only if $\lambda_{1} \simeq \lambda_{0} \simeq \lambda_{\infty}$.

The next result is a consequence of this result and the former proposition.

Corollary 2.2. Given a real number $p$ with $p>1, \lambda_{p}$ is a nuclear space if and only if $\lambda_{p} \simeq \lambda_{0} \simeq \lambda_{\infty}$.

We need the following lemma essentially included in [6].

Lemma (Dvoretzky-Rogers). Let $(E,\|\|)$ be an infinite dimensional normed space and let $\delta=\left(\delta_{j}\right)$ be an element of $l^{2}$. Then there is $x=\left(x_{j}\right)$ in $l^{1}(E)$ with $\left\|x_{j}\right\|=\left|\delta_{j}\right|$ for all $j$ in $\mathbb{N}$.

Theorem 2.3. Let $(E,\|\|)$ be a normed space and let $p$ be a real number with $p>1$, the following are equivalent:

(i) $\lambda_{p}(E) \simeq \lambda_{p}\{E\}$

(ii) $\lambda_{p}[E] \simeq \lambda_{p}\{E\}$

(iii) $\lambda_{p} \otimes_{e} E \simeq \lambda_{p} \otimes_{\pi} E$,

(iv) $\lambda_{p} \widetilde{\otimes}_{e} E \simeq \lambda_{p} \tilde{\otimes}_{\pi} E$,

(v) $\lambda_{p}$ is a nuclear space or $E$ is finite dimensional.

Proof. (i) $\Rightarrow$ (ii): Since $\lambda_{p}(E)$ is provided with the $\varepsilon$-topology and $\lambda_{p}\{E\} \subset \lambda_{p}(E) \subset \lambda_{p}[E]$, we need only to show that $\lambda_{p}[E] \subset \lambda_{p}\{E\}$. Let $x=\left(x_{j}\right)$ be an element in $\lambda_{p}[E]$. Then every section $x^{(n)}$ of $x$ is in $\lambda_{p}(E)$. By hypothesis, given a positive integer $r$ there is a positive integer $k>r$ and a real number $\rho>0$ such that

$$
\begin{aligned}
\rho \pi_{r}\left(x^{(n)}\right) & =\rho\left(\sum_{j=1}^{n}\left(\left\|x_{j}\right\| \cdot a_{j}^{(r)}\right)^{p}\right)^{1 / p} \\
& \leqq \sup \left\{\left(\sum_{j=1}^{n}\left|\left\langle x_{j}, u\right\rangle a_{j}^{(k)}\right|^{p}\right)^{1 / p}: u \in U^{0}\right\} \\
& =\varepsilon_{k}\left(x^{(n)}\right),
\end{aligned}
$$


where $U^{0}$ is the polar set in $E^{\prime}$ of the closed unit ball $U$ of $E$. Since this hold for all $n$, we obtain

$$
\rho \pi_{r}(x) \leqq \varepsilon_{k}(x)<\infty
$$

(ii) $\Rightarrow$ (i) is obvious. Also (iii) $\Leftrightarrow$ (ii) is clear and (v) $\Rightarrow$ (iii) is a well known result (see [12] 7.3.3). (iii) $\Rightarrow$ (i) follows by an argument similar to [10] p. 197 and p. 291.

(i) $\Rightarrow(v)$ : We suppose $E$ is infinite dimensional. Given a positive integer $r$, by (i), there is a positive integer $k>r$ and a real number $\rho>0$ such that $\rho \pi_{r}(x) \leqq \varepsilon_{k}(x)$ for all $x$ in $\lambda_{p}(E)$, i.e.,

$$
\rho\left(\sum_{j=1}^{\infty}\left(\left\|x_{j}\right\| a_{j}^{(r)}\right)^{p}\right)^{1 / p} \leqq \sup \left\{\left(\sum_{j=1}^{\infty}\left|\left\langle x_{j}, u\right\rangle a_{j}^{(k)}\right|^{p}\right)^{1 / p}: u \in U^{0}\right\} \text { for all } x \in \lambda_{p}(E) .
$$

Let $y=\left(y_{j}\right)$ be an element in $l^{1}(E)$. Define $y^{n}=\left(y_{j}^{n}\right)$ for every $n$ in $\mathbb{N}$, such that

$$
y_{j}^{n}=\left(y_{j},\left\|y_{j}\right\|^{1 / q} a_{j}^{(k)}\right) \quad \text { if } j \leqq n
$$

$y_{j}^{n}=0$ if $j>n, q$ being the conjugate number of $p$. Since $y^{n}$ belongs to $\lambda_{p}(E)$ for all $n$, it follows from (1) that

$$
\begin{aligned}
\rho\left(\sum_{j=1}^{n}\left(a_{j}^{(r)} ; a_{j}^{(k)}\right)^{p}\left\|y_{j}\right\|\right)^{1 / p} & \leqq \sup \left\{\left(\sum_{j=1}^{n}\left(\left|\left\langle y_{j}, u\right\rangle\right|^{p} ;\left\|y_{j}\right\|^{p / q}\right)\right)^{1 / p}: u \in U^{0}\right\} \\
& \leqq \sup \left\{\left(\sum_{j=1}^{\infty}\left|\left\langle y_{j}, u\right\rangle\right|\right)^{1 / p}: u \in U^{0}\right\}
\end{aligned}
$$

and consequently

$$
\rho\left(\sum_{j=1}^{\infty}\left(a_{j}^{(r)} ; a_{j}^{(k)}\right)^{p}\left\|y_{j}\right\|\right)<\infty \quad \text { for all } \quad y \in l^{1}(E)
$$

Applying the Dvoretzky-Rogers lemma to (3), we get that

$$
\left(\left(a_{j}^{(r)} ; a_{j}^{(k)}\right)^{p}\right)
$$

is an element of $l^{2}$.

Repeating the former argument for $k$ and applying Holder's inequality, we can state that, given a positive integer $r$, there is a positive integer $s>r$ such that

$$
\left(a_{j}^{(r)} ; a_{j}^{(s)}\right)
$$

is in $l^{p}$, so by $2.1, \lambda_{p}$ is nuclear. 


\section{The Dvoretzky-Rogers Theorem for echelon spaces of order $(p, q)$}

Let $\left\{a^{(r)}=\left(a_{i j}^{(r)}\right)\right\}$ be a sequence of elements of $\omega$ satisfying:

(i) $a_{i j}^{(r)}>0$ for all $r, i, j \in \mathbb{N}$

(ii) $a_{i j}^{(r)} \leqq a_{i j}^{(r+1)}$ for all $r, i, j \in \mathbb{N}$.

If $p$ and $q$ are real numbers with $p \geqq 1$ and $q \geqq 1$, we denote by $\Lambda_{p q}$ the echelon space of order $(p, q)$ defined by the steps $\left\{a^{(r)}\right\}$ (see $\left.[1]\right)$, i.e.,

$$
\Lambda_{p q}=\left\{x=\left(x_{i j}\right) \in \omega: \sum_{j=1}^{\infty}\left(\sum_{i=1}^{\infty}\left|x_{i j} a_{i j}^{(r)}\right|^{p}\right)^{q / p}<\infty \text { for all } r \in \mathbb{N}\right\}
$$

endowed with the topology defined by the seminorms:

$$
\|x\|_{r}=\left(\sum_{j=1}^{\infty}\left(\sum_{i=1}^{\infty} \mid x_{i j} a_{i j}^{(r) \mid p}\right)^{q / p}\right)^{1 / p}, r \in \mathbb{N} .
$$

Analogously, we denote by $\lambda_{\infty}$ the echelon space of infinite order defined by the steps $\left\{a^{(r)}\right\}$ and provided with the topology defined by the seminorms:

$$
|x|_{r}=\sup _{i, j}\left|x_{i j} a_{i j}^{(r)}\right|, r \in \mathbb{N} .
$$

From now on $p$ and $q$ will denote conjugate real numbers with $p>1$ and $q>1$.

In [1] we proved the following result:

Proposition 3.1. The space $\Lambda_{p q}$ is nuclear if and only if, given a positive integer $r$, there is a positive integer $k>r$ such that

$$
\sum_{i, j=1}^{\infty}\left(a_{i j}^{(r)} ; a_{i j}^{(k)}\right)<\infty
$$

By this proposition and since

$$
\Lambda_{p q} \subset \Lambda_{p p} \subset \lambda_{\infty} \quad \text { or } \quad \Lambda_{p q} \subset \Lambda_{q q} \subset \lambda_{\infty}
$$

we get:

Corollary 3.2. The space $\Lambda_{p q}$ is nuclear if and only if

$$
\Lambda_{p q} \simeq \lambda_{\infty}
$$

We can now establish the following useful characterization of nuclearity:

Proposition 3.3. The space $\Lambda_{p q}$ is muclear if and only if given a positive integer $r$ there is a positive integer $k>r$ such that 


$$
\sup _{j}\left(\sum_{i=1}^{\infty}\left(a_{i j}^{(r)} ; a_{i j}^{(k)}\right)^{p}\right)<\infty
$$

and

$$
\sum_{j=1}^{\infty}\left(\sup _{i}\left(a_{i j}^{(r)} ; a_{i j}^{(k)}\right)\right)^{q}<\infty
$$

Proof. If $\Lambda_{p q}$ is a nuclear space it follows from Proposition 3.1 that the condition (1) is satisfied.

Conversely, suppose that condition (1) is satisfied. We consider the space

$$
\Lambda_{1}=\left\{x=\left(x_{i j}\right) \in \omega: \sum_{j=1}^{\infty}\left(\sup _{i}\left|x_{i j} a_{i j}^{(r)}\right|\right)^{q}<\infty \text { for all } r \in \mathbb{N}\right\}
$$

provided with the topology defined by the seminorms:

$$
\|x\|_{r}^{1}=\left(\sum_{i=1}^{\infty}\left(\sup _{i}\left|x_{i j} a_{i j}^{(r)}\right|\right)^{q}\right)^{1 / q}, r \in \mathbb{N} .
$$

Let $x$ be an element of $\Lambda_{p q}$, then $x$ belongs to $\Lambda_{1}$ and $\|x\|_{r}^{1} \leqq\|x\|_{r}$ for all $r$ in $\mathbb{N}$. On the other hand, if $x$ is an element of $\Lambda_{1}$, given a positive integer $r$ we suppose there exists a positive integer $k>r$ such that (1) is satisfied. Then

$$
\begin{aligned}
\|x\|_{r} & \leqq\left(\sum_{j=1}^{\infty}\left(\sup _{i}\left|x_{i j} a_{i j}^{(k)}\right|\right)^{q}\left(\sum_{i=1}^{\infty}\left(a_{i j}^{(r)} ; a_{i j}^{(k)}\right)^{p}\right)^{q / p}\right)^{1 / q} \\
& \leqq\left(\sup _{j}\left(\sum_{i=1}^{\infty}\left(a_{i j}^{(r)} ; a_{i j}^{(k)}\right)^{p}\right)^{1 / p}\right)\|x\|_{k}^{1}<\infty
\end{aligned}
$$

from where it follows that $\Lambda_{p q} \simeq \Lambda_{1}$.

Analogously, it is easy to prove that $\Lambda_{1} \simeq \lambda_{\infty}$. Finally, applying 3.2 , we obtain that $\Lambda_{p q}$ is a nuclear space.

Theorem 3.4. Let $(E,\|\|)$ be a normed space. The following are equivalent:

(i) $\Lambda_{p q}(E) \simeq \Lambda_{p q}\{E\}$,

(ii) $\Lambda_{p q}[E] \simeq \Lambda_{p q}\{E\}$,

(iii) $\Lambda_{p q} \otimes{ }_{\varepsilon} E \simeq \Lambda_{p q} \otimes_{\pi} E$,

(iv) $\Lambda_{p q} \widetilde{\otimes}_{\varepsilon} E \simeq \Lambda_{p q} \widetilde{\otimes}_{\pi} E$,

(v) $\Lambda_{p q}$ is a nuclear space or $E$ is finite dimensional.

Proof. As in the proof of 2.3 , it is easy to prove that (i) $\Leftrightarrow$ (ii), (iii) $\Leftrightarrow$ (iv), (v) $\Rightarrow$ (iii) and (iii) $\Rightarrow$ (i). We now prove (i) $\Rightarrow(v)$. Suppose that $E$ is infinite dimensional. Since $\Lambda_{p q}(E) \simeq \Lambda_{p q}\{E\}$ by assumption, given a positive integer $r$ there is a positive integer $k>r$ 
and a real number $\rho>0$ such that $\rho \pi_{r}(x) \leqq \varepsilon_{k}(x)$ for all $x$ in $\Lambda_{p q}(E)$, i.e.,

$$
\rho\left(\sum_{j=1}^{\infty}\left(\sum_{i=1}^{\infty}\left(\left\|x_{i j}\right\| a_{i j}^{(r)}\right)^{p}\right)^{q / p}\right)^{1 / q} \leqq \sup \left\{\left(\sum_{j=1}^{\infty}\left(\sum_{i=1}^{\infty}\left|\left\langle x_{i j}, u\right\rangle a_{i j}^{(k)}\right|^{p}\right)^{q / p}\right)^{1 / q}: u \in U^{0}\right\}
$$

for all $x=\left(x_{i j}\right)$ in $\Lambda_{p q}(E), U^{0}$ being the polar set in $E^{\prime}$ of the closed unit ball $U$ of $E$.

We denote by $\Delta(E)$ the subspace of $\omega(E)$ of all the elements $x=\left(x_{i j}\right)$ such that

$$
\sup \left\{\left(\sum_{j=1}^{\infty}\left(\sum_{i=1}^{\infty}\left|\left\langle x_{i j}, u\right\rangle\right|\right)^{q / p}\right): u \in U^{0}\right\}<\infty .
$$

Given $x=\left(x_{i j}\right)$ in $\Delta(E)$, define $y^{n}=\left(y_{i j}^{n}\right)$ for every $n$ in $\mathbb{N}$ by

$$
\begin{aligned}
& y_{i j}^{n}=\left(x_{i j} ;\left\|x_{i j}\right\| \|^{1 / q} a_{i j}^{(k)}\right) \text { if } i \leqq n \text { and } j \leqq n \\
& y_{i j}^{n}=0 \text { if } i>n \text { or } j>n .
\end{aligned}
$$

By (1), we have:

$$
\begin{aligned}
\rho \pi_{r}\left(y^{n}\right) & =\rho\left(\sum_{j=1}^{n}\left(\sum_{i=1}^{n}\left(a_{i j}^{(r)} ; a_{i j}^{(k)}\right)^{p}\left\|x_{i j}\right\|\right)^{q / p}\right)^{1 / q} \\
& \leqq \sup \left\{\left(\sum_{j=1}^{n}\left(\sum_{i=1}^{n}\left(\left|\left\langle x_{i j}, u\right\rangle\right|^{p} ;\left\|x_{i j}\right\|^{p / q}\right)\right)^{q / p}\right)^{1 / q}: u \in U^{0}\right\} \\
& =\varepsilon_{k}\left(y^{n}\right) \leqq \sup \left\{\left(\sum_{j=1}^{\infty}\left(\sum_{i=1}^{\infty} \mid\left\langle x_{i j}, u\right\rangle\right)^{q / p}\right)^{1 / q}: u \in U^{0}\right\}
\end{aligned}
$$

from where it follows that

$$
\begin{gathered}
\rho\left(\sum_{j=1}^{\infty}\left(\sum_{i=1}^{\infty}\left(a_{i j}^{(r)} ; a_{i j}^{(k)}\right)^{p}\left\|x_{i j}\right\|\right)^{q / p}\right)^{1 / q} \\
\leqq \sup \left\{\left(\sum_{j=1}^{\infty}\left(\sum_{i=1}^{\infty}\left|\left\langle x_{i j}, u\right\rangle\right|\right)^{q / p}\right)^{1 / q}: u \in U^{0}\right\} \text { for all } x \in \Delta(E) .
\end{gathered}
$$

Let $y=\left(y_{i}\right)$ be an element of $l^{1}(E)$. Define $z^{j}=\left(z_{i k}^{j}\right)$ for every $j$ in $\mathbb{N}$, such that

$$
z_{i j}^{j}=y_{i}, z_{i k}^{j}=0 \text { for all } k \neq j, \quad i=1,2, \ldots
$$

Obviously, $z^{j}$ is in $\Delta(E)$ and it follows from (3) that

$$
\begin{gathered}
\rho\left(\sum_{i=1}^{\infty}\left(a_{i j}^{(r)} ; a_{i j}^{(k)}\right)^{p}\left\|y_{i}\right\|\right)^{1 / p} \\
\leqq \sup \left\{\left(\sum_{i=1}^{\infty}\left|\left\langle y_{i}, u\right\rangle\right|\right)^{1 / p}: u \in U^{0}\right\} \text { for all } y \in l^{1}(E), j \in \mathbb{N} .
\end{gathered}
$$


Applying the Dvoretzky-Rogers lemma to (4), we obtain that

$$
\left\{\left(\left(a_{i j}^{(r)} ; a_{i j}^{(k)}\right)^{p}\right)_{i}, j \in \mathbb{N}\right\}
$$

is a bounded subset of $l^{2}$.

Repeating the former argument for $k$ and using Holder's inequality, we can suppose that, given a positive integer $r$, there is a positive integer $s>r$ with

$$
\sup _{j}\left(\sum_{i=1}^{\infty}\left(a_{i j}^{(r)} ; a_{i j}^{(s)}\right)^{p}\right)<\infty
$$

We consider the space $\Lambda_{1}$ defined in 3.3. As in the proof of this proposition, we can conclude that $\Lambda_{1} \simeq \Lambda_{p q}$. Therefore $\Lambda_{1}(E) \simeq \Lambda_{1}\{E\}$ and consequently, given a positive integer $r$, there is a positive integer $k>r$ and $h>0$ such that

$$
\begin{gathered}
h\left(\sum_{j=1}^{\infty}\left(\sup _{i}\left(\left\|x_{i j}\right\| a_{i j}^{(r)}\right)^{q}\right)\right)^{1 / q} \\
\leqq \sup \left\{\left(\sum_{j=1}^{\infty}\left(\sup _{i}\left|\left\langle x_{i j}, u\right\rangle a_{i j}^{(k)}\right|^{q}\right)\right)^{1 / q}: u \in U^{0}\right\} \text { for all } x \in \Lambda_{1}(E) .
\end{gathered}
$$

Let $y=\left(y_{j}\right)$ be an element of $l^{1}(E)$, for every $n$ in $\mathbb{N}$, we define $x^{n}=\left(x_{i j}^{n}\right)$ by

$$
\begin{aligned}
& x_{i j}^{n}=\left(y_{j} ;\left\|y_{j}\right\|^{1 / p} a_{i j}^{(k)}\right) \text { if } i \leqq n \text { and } j \leqq n \\
& x_{i j}^{n}=0 \text { if } i>n \text { or } j>n .
\end{aligned}
$$

Hence $x^{n} \in \Lambda_{1}(E)$ for all $n$ in $\mathbb{N}$. Now it follows from (6) that

$$
\begin{aligned}
h\left(\sum_{j=1}^{n}\left\|y_{j}\right\| \cdot\left(\sup _{i=1}^{n}\left(a_{i j}^{(r)} ; a_{i j}^{(k)}\right)^{q}\right)\right)^{1 / q} & \leqq \sup \left\{\left(\sum_{j=1}^{n}\left(\left|\left\langle y_{j}, u\right\rangle\right|^{q} ;\left\|y_{j}\right\|^{q / p}\right)\right)^{1 / q}: u \in U^{0}\right\} \\
& \leqq \sup \left\{\left(\sum_{j=1}^{\infty} \mid\left\langle y_{j}, u\right\rangle\right)^{1 / q}: u \in U^{0}\right\},
\end{aligned}
$$

and consequently

$$
h\left(\sum_{j=1}^{\infty}\left\|y_{j}\right\| \cdot\left(\sup _{i}\left(a_{i j}^{(r)} ; a_{i j}^{(k)}\right)^{q}\right)\right)^{1 / q} \leqq \sup \left\{\left(\sum_{j=1}^{\infty}\left|\left\langle y_{j}, u\right\rangle\right|\right)^{1 / q}: u \in U^{0}\right\} \text { for all } y \in l^{1}(E) .
$$

Proceeding in the same way as we did to obtain (5), we establish that, given a positive integer $r$, there is a positive integer $s>r$ such that

$$
\sum_{j=1}^{\infty}\left(\sup _{i}\left(a_{i j}^{(r)} ; a_{i j}^{(s)}\right)\right)^{q}<\infty
$$

Finally, from (5), (7) and 3.3, it follows that $\Lambda_{p q}$ is nuclear. 
Acknowledgment. This paper, with minor alterations, is part of the author's Ph.D. Dissertation, "Algunos nuevos resultados en espacios de sucesiones", written at the University of Valencia under the direction of Professor M. Valdivia, whom I wish to thank for his helpful direction.

\section{REFERENCES}

1. F. Andreu, Espacios escalonados de orden $(p, q)$. (to appear).

2. H. Apiola, Duality between spaces of $p$-summable sequences, $(p, q)$-summing operators and characterizations of nuclearity, Math. Ann. 64 (1976), 53-64. 166.

3. N. de Grande de Kimpe, Generalized sequence spaces, Bull. Soc. Math. Belg. 23 (1981), 123-

4. N. DE Grande de Kimpe, Locally convex spaces for which $\Lambda(E)=\Lambda[E]$ and the DvoretzkyRogers theorem, Compositio Math. 35 (1977), 139-145.

5. E. Dubinsky, Echelon spaces of order $\infty$, Proc. Amer. Math. Soc. 16 (1965), 1178-1183.

6. A. Dvoretzky and C. Rogers, Absolute and unconditional convergence in normed linear spaces, Proc. Nat. Acad. Sci. U.S.A. 36 (1950), 192-197.

7. A. Grothendieck, Sur certains classes de suites dans les espaces de Banach et le théorème de Dvoretzky-Rogers, Bol. Soc. Math. Sao Paulo 8 (1956), 81-110.

8. H. JarChow, Locally Convex Spaces (Stuttgart: B. G. Teubner, 1981).

9. G. KöтнE, Topological Vector Spaces I (Berlin-Heidelberg-New York: Springer, 1969).

10. G. Котне, Topological Vector Spaces II (Berlin-Heidelberg-New York: Springer, 1980).

11. A. Piftsch, Veralgemeinerte volkommene Folgen Räume (Akademie Verlag, 1962).

12. A. Pietsch, Nuclear Locally Convex Spaces (Berlin-Heidelberg-New York: Springer, 1972).

13. R. C. Rosier, Dual spaces of certain vector sequence spaces, Pacific J. Math. 46 (1973), 487501.

14. R. C. Rosier, Vector sequence spaces and the Dvoretzky-Rogers theorem (to appear).

15. M. VALDIVIA, Topics in locally convex spaces (Amsterdam-New York-Oxford: Mathematics Studies 67, 1982).

Facultad de Matemáticas

Dr. MOLINER, 50

BurJasot (VALENCIA)

SPAIN 